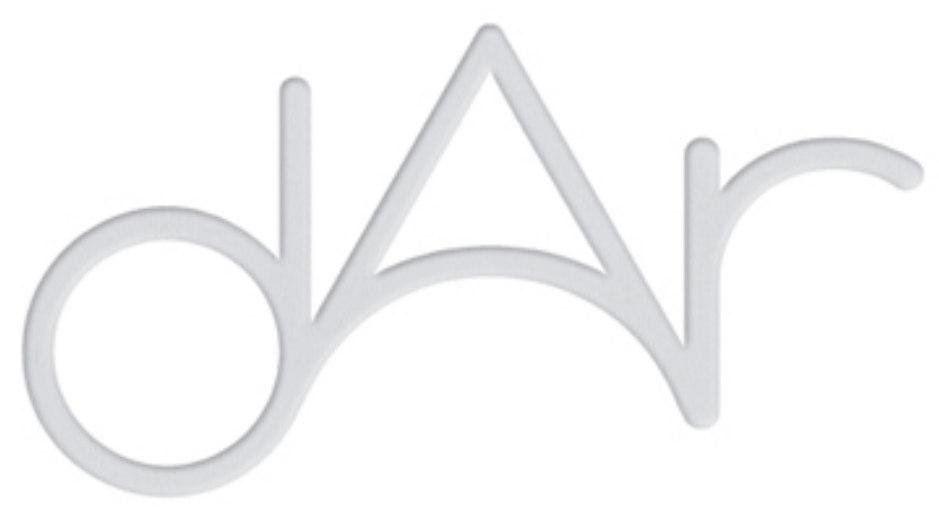

\title{
Architecture traditionnelle dans les zones de montagne: contribution à l'étude de la typologie des habitations dans le Haut Atlas au Maroc
}

Autor(es): $\quad$ Costa, Miguel Reimão; Batista, Desidério

Publicado por: Imprensa da Universidade de Coimbra

URL

persistente: URI:http://hdl.handle.net/10316.2/44442

DOI: $\quad$ DOl:https://doi.org/10.14195/2182-844X_5_21

Accessed : $\quad$ 26-Apr-2023 16:36:43

A navegação consulta e descarregamento dos títulos inseridos nas Bibliotecas Digitais UC Digitalis, UC Pombalina e UC Impactum, pressupõem a aceitação plena e sem reservas dos Termos e Condições de Uso destas Bibliotecas Digitais, disponíveis em https://digitalis.uc.pt/pt-pt/termos.

Conforme exposto nos referidos Termos e Condições de Uso, o descarregamento de títulos de acesso restrito requer uma licença válida de autorização devendo o utilizador aceder ao(s) documento(s) a partir de um endereço de IP da instituição detentora da supramencionada licença.

Ao utilizador é apenas permitido o descarregamento para uso pessoal, pelo que o emprego do(s) título(s) descarregado(s) para outro fim, designadamente comercial, carece de autorização do respetivo autor ou editor da obra.

Na medida em que todas as obras da UC Digitalis se encontram protegidas pelo Código do Direito de Autor e Direitos Conexos e demais legislação aplicável, toda a cópia, parcial ou total, deste documento, nos casos em que é legalmente admitida, deverá conter ou fazer-se acompanhar por este aviso. 

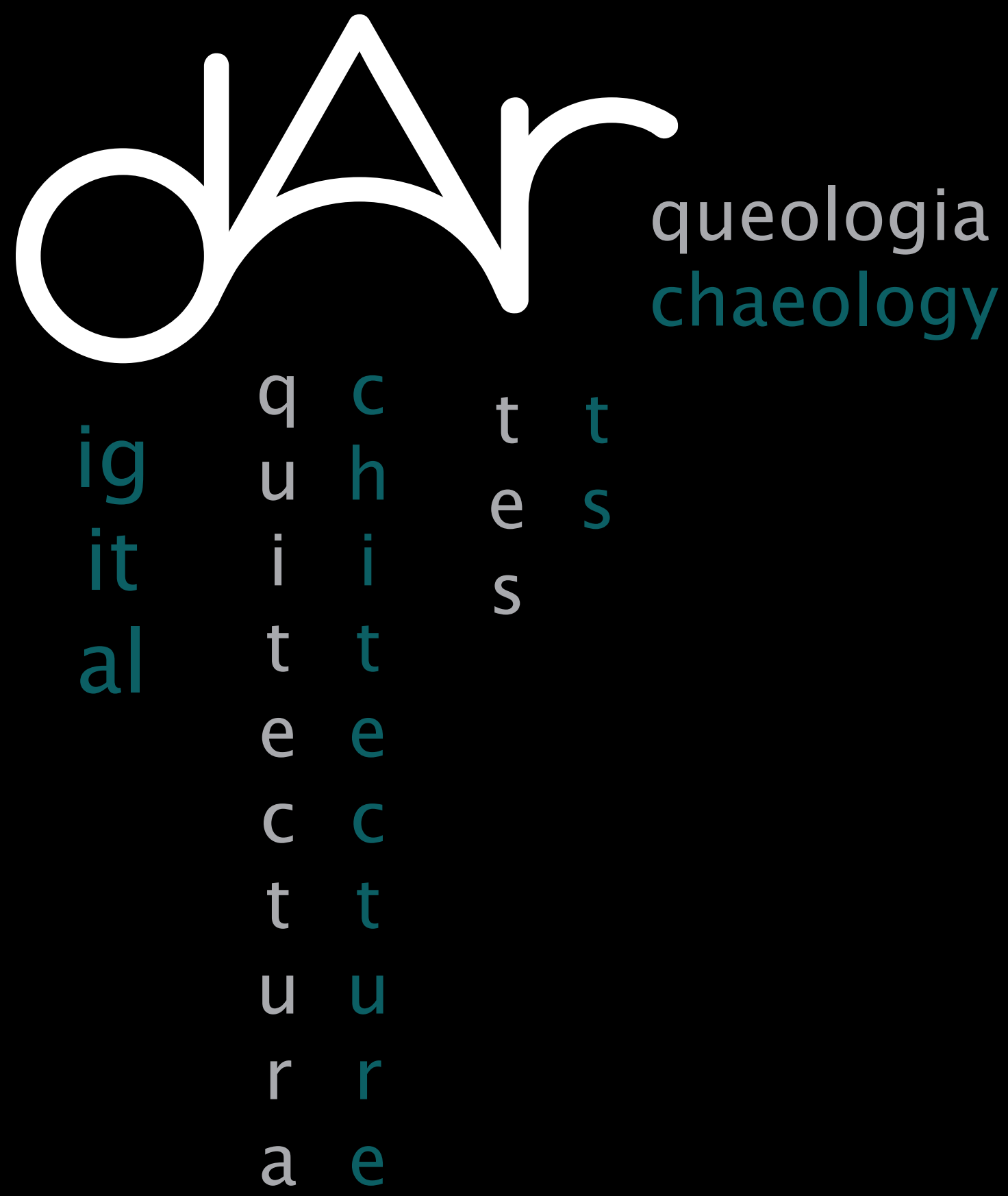

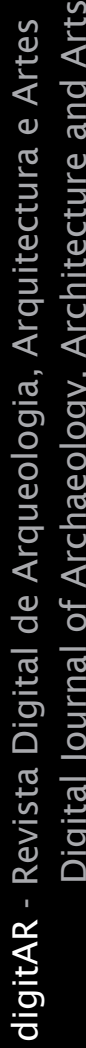


ArChitecture traditionNelle DaNS LeS zones De MONTAGNE: CONTRIBUtion À L'étude de la typologie des habitations dans le Haut Atlas au Maroc

Miguel Reimão Costa

CEAACP, Université de l'Algarve, mrcosta@ualg.pt

Desidério Batista

CHAIA, Université de l'Algarve, $\underline{\text { dbatista@ualg.pt }}$

\section{Introduction}

Le système montagneux du Haut Atlas marocain comprend diverses sous-unités de paysage dont l'identité provient de l'interaction entre facteurs naturels (géologie, relief, sols, climat, hydrologie, végétation) et facteurs culturels qui caractérisent la société tribale berbère. Une extension géographique notable de cette région montagneuse et sa large amplitude altimétrique, associées à une grande diversité géologique, morphologique, climatique et floristique, donnent lieu à divers habitats ruraux et à différents systèmes de production agro-pastorale. En effet, en raison d'une adaptation constante à des difficiles conditions naturelles, les groupes sociaux de type tribal ont construit un modèle historique d'occupation et d'organisation territoriale, fondé sur le traditionnel système agro-sylvo-pastoral comme base d'une économie de subsistance. Ce modèle et ce système sont marqués par les caractéristiques biophysiques du territoire montagneux et par la complémentarité entre champs irrigués (urti) dans les vallées et champs secs (bour) sur les versants les moins pentus, pâturages sur les hautes terres et forêts aux diverses espèces d'arbres, sur les versants situés en altitude et présentant une déclivité variable.

De là résulte un modèle de sous-unités de paysage liées au système de peuplement de montagne par excellence, à savoir la relation entre les villages (principaux et secondaires), reflet des différentes formes de transhumance associée à l'élevage et de sédentarisation associée à l'agriculture. De toute façon, la diversité qui caractérise les différents territoires du Haut Atlas se retrouve de manière expressive dans les différents modèles d'organisation qui sont lisibles depuis le peuplement jusqu'à l'habitation. La présente étude porte sur l'analyse de l'architecture vernaculaire du Haut Atlas,

https:/ /doi.org/10.14195/2182-844X_5_21 
réunissant quelques-uns des thèmes qui caractérisent sa diversité, dans le but de contribuer à l'établissement d'une carte typologique. Sur le plan méthodologique, on privilégie ici la caractérisation diatopique par rapport à la lecture diachronique, en cherchant à croiser le travail de terrain, réalisé à partir d'études fondamentales sur le territoire, la culture et l'architecture traditionnelle de ces régions. Pour cela, nous considérerons, dans un premier temps, les différents contextes et sous-unités de paysages du Haut Atlas, réunissant un éventail de thèmes qui vont du territoire physique aux ressources disponibles, avec les implications au niveau des procédés de construction traditionnels. Dans un second temps, seront analysées différentes habitations de caractère permanent, localisées dans les sousunités géographiques citées, qui ont été relevées et dessinées par nos soins dans le cadre de cette recherche ${ }^{1}$.

Dans une conclusion, on cherchera à tracer une brève synthèse de cette architecture dans sa relation avec le paysage, à partir des exemples analysés antérieurement, comme contribution à une carte ty pologique de l'architecture traditionnelle du Haut Atlas.

\section{Pour une lecture de la diversité dans le Haut Atlas}

Sur ce territoire montagneux, en allant de l'est vers l'ouest et en considérant la situation sur le versant nord ou sur le versant sud du ou des bassins hydrographiques, on a relevé de grandes unités spatiales qui reflètent différentes façons d'habiter et de produire en fonction des ressources disponibles et des techniques culturales ancestrales. La Carte Structurale du Maroc (Saidi, 1982), la Carte des centres à haut taux d'endémisme en Méditerranée (Quézel, 1995), la Carte Agricole et la Carte des Tribus du Maroc mettent l'accent sur l'importante diversité géologique, sur la présence significative d'espèces végétales autochtones (taux d'endémisme supérieur à $20 \%$ ) et sur la grande diversité socioculturelle, liées aux différentes

\footnotetext{
1 La présente recherche s'inscrit dans L'Etude intégrée de l'architecture et du paysage des régions de montagne de la Méditerranée occidentale, financée par Fundação para a Ciência e a Tecnologia (SFRH/BSAB/114338/2016 e SFRH/BSAB/114311/2016), relative au premier semestre de 2016, pour l'élaboration du travail de terrain au Maroc, intégré au LERMA-TDD, Laboratoire Les Montagnes Atlasiques - Territoires, Développement et Durabilité, Université Cadi Ayyad, Marrakech. Tous les dessins présentés ont été réalisés à partir de relevés exécutés dans le cadre de cette recherche. Les auteurs remercient José Alberto Alegria, João Guerreiro, Maria Ramalho, Salima Naji, Ahmed Ouhammou et Said Boujrouf.
} 
sous-régions montagneuses et aux différentes expressions d'architecture vernaculaire qui les caractérise. On relève la diversité des solutions architectoniques identifiées qui ont toujours recourt aux matériaux locaux, en utilisant différents types de terre, de pierre et de bois et aboutissant à une totale intégration dans le paysage (Fig. 1). La fusion entre l'architecture et le paysage dans chacune de ces sous-régions montagneuses se remarque, entre autres, dans l'implantation de la maison, dans sa volumétrie et dans les procédés et les matériaux de construction utilisés, aussi bien en ce qui concerne la pierre et la terre que le bois. Les différentes architectures reflètent exactement la diversité géologique et la diversité de la flore de chaque sousunité paysagère, en recourant pour chacune soit à différents types de roche et de terre, soit à des espèces d'arbre, d'arbuste et de buisson les plus aptes pour les multiples fonctions dans la construction: structurales, décoratives, de revêtement, de protection, etc., selon l'utilisation de savoirs et de techniques traditionnels.

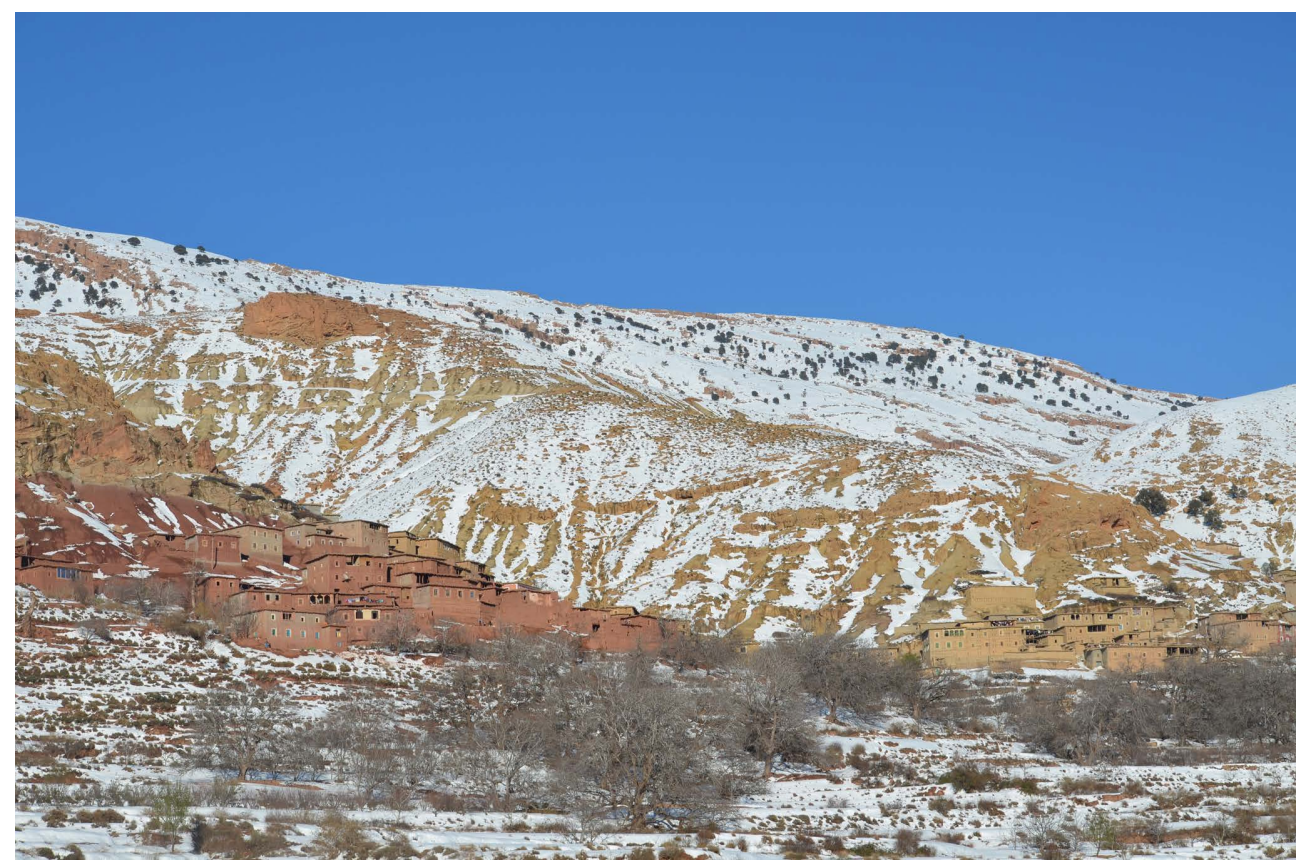

Fig. 1

Dans le Haut Atlas oriental, la diversité du substrat rocheux (calcaire, schiste, diorite, syénite, basalte, entre autres) et les terres qui lui sont associées sont 
utilisées dans les différents procédés de construction de la maison. A côté de l'importance de la pierre, du pisé et de l'adobe, le bois de cèdre surgit comme un élément fondamental dans l'architecture domestique. Ici, dans les montagnes recouvertes de forêts discontinues de cèdres «[...] ces bâtisses de pisé ont recueilli l'héritage d'une ancienne architecture de bois [...]» (Terrasse, 1938: 107). Dans la transition vers l'Atlas central, sur le versant nord du système montagneux qui, en fonction de l'altitude, est couvert par une forêt dense de chênes verts, dans les niveaux les plus bas, et d'une forêt discontinue de cèdres, à un niveau supérieur, on constate la prédominance de roches sédimentaires comme les dolomies, les marnes et parfois les grès (toutefois avec des plaques intrusives de gabro-diorites), qui marquent l'architecture de façon indélébile. Celle-ci montre des caractéristiques distinctes dans des sous-unités de paysage de l'Atlas central, associées au versant nord de la Jbel $\mathrm{M}^{\prime}$ goun (4071 mètres) dont la diversité géologique se remarque par la présence de calcaire, grès, schistes, basaltes doléritiques et granites et dont la diversité de la flore comprend différentes espèces d'arbres, arbustes et buissons, certaines endémiques (Ouhammou, 2005: 8). Parmi ces espèces, un bois assume un rôle particulièrement évident, $c^{\prime}$ est le bois de genévrier thurifère qui, abondamment utilisé aussi dans la cuisine, le chauffage et comme fourrage foliaire, se trouve en danger d 'extinction car sa régénération naturelle est très difficile (Ouhammou; Muller; M'Hirit, 1996). Il convient encore de signaler, pour son utilisation particulière à l'extrémité des couvertures planes des édifices, d'une espèce de buisson Cladanthus scarosus (Ball.) Oberpr.\&Vogt.

Dans la transition vers le Haut Atlas occidental, les reliefs deviennent chaque fois plus vigoureux; dans sa partie ouest, on remarque la Jbel Toubkal (4165 mètres) où se détachent deux unités de paysage sur les versants, nord et sud. Ces unités se différencient non seulement par la géologie, nettement différente dans chacune, mais surtout par l'influence des vents océaniques humides associés à une plus grande pluviosité sur les versants nord et, au contraire, par l'influence de vents secs et chauds du sud, associés à une plus grande aridité et sécheresse sur les versants méridionaux. A cette opposition climatique correspond une différence de la flore et de la végétation qui, en fonction de l'altitude également (Cf. Bellaoui, 1986), présentent quelques traits distinctifs: sur le versant méridional, l'arganier et le dattier poussent à des altitudes assez supérieures à ce que l'on constate sur le versant nord. Sur les deux côtés de la ligne de faite, on enregistre, en fonction de l'altitude, comme le signale Bellaoui (1986), la répartition des espèces suivantes: le thuya entre 500 et 1200 mètres, le chêne vert, entre cette limite et près de 2000 mètres, puis jusqu'à 2500 mètres, le genévrier thurifère (Fig. 2). Le travail de terrain 
a révélé que, en fonction de la distribution des espèces d'arbre selon les différents versants et selon les différentes altitudes et en fonction des caractéristiques des bois respectifs, on a utilisé pour la construction des maisons, de façon plus ou moins intensive et dans les éléments structuraux ou complémentaires, le bois d'arganier et de dattier dans les habitations de moyenne altitude des versants sud du Haut Atlas occidental, et on a recouru de façon privilégiée au bois de genévrier dans les constructions des niveaux supérieurs.
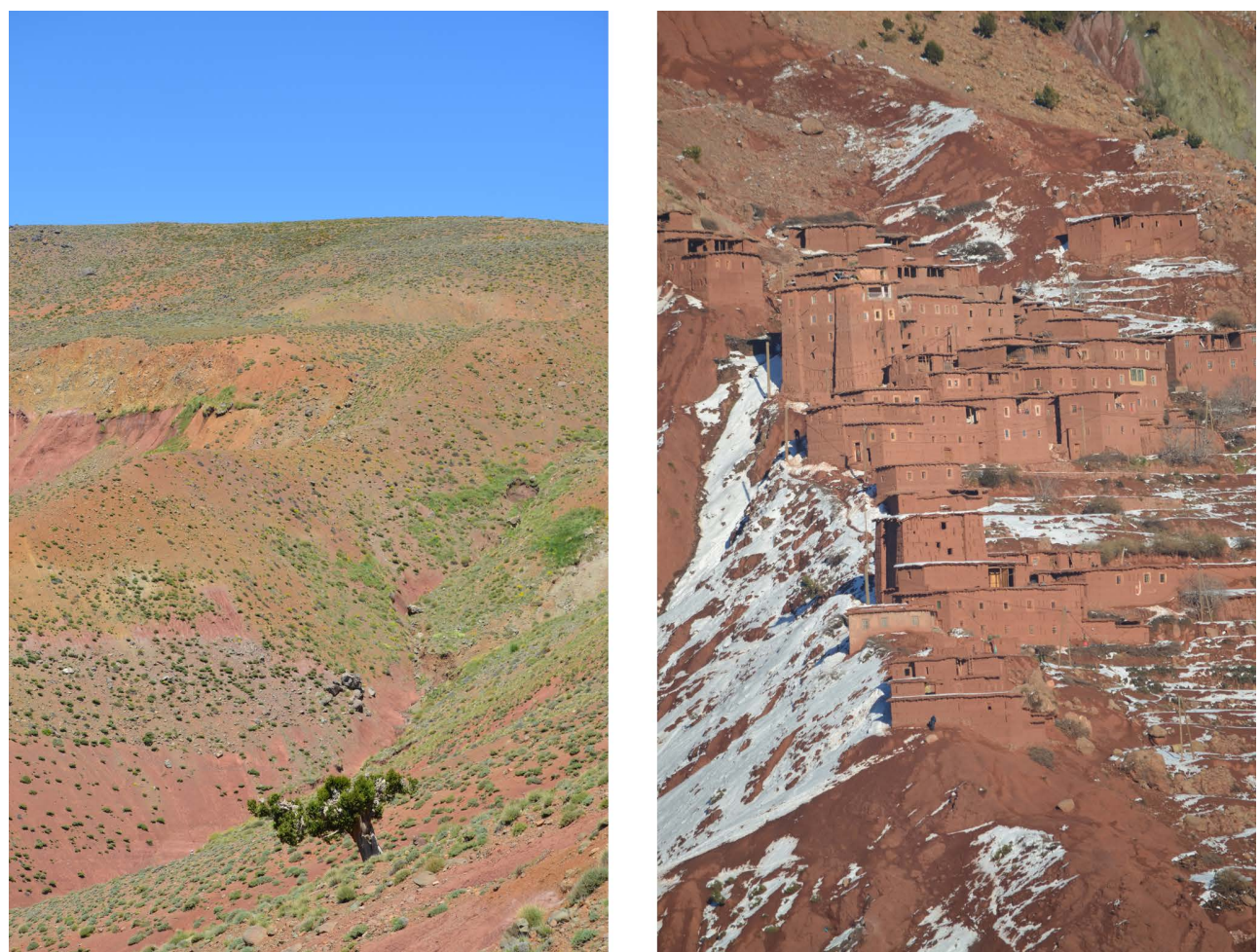

Fig. 2

\section{Les maisons relevées dans les différentes unités de paysage}

L'étude de l'architecture traditionnelle est systématiquement mise en situation par l'importance des circonstances et du lieu. Dans le Haut Atlas, il est primordial de comprendre la façon dont la diversité abiotique, biotique et culturelle, qui caractérise ses diverses sous-unités, transparaît au niveau de l'habitat et de l'architecture domestique. En général, nous pouvons affirmer 
que, de même que le peuplement concentré en petits villages constitue la forme privilégiée de l'habitat de la région, de même l'habitation, avec un ou deux étages au-dessus des dépendances destinées au bétail et au fourrage, constitue, dans ses diverses variantes, la solution commune dans cette région montagneuse. Comme cela apparaît dans la plupart des zones de montagne de la Méditerranée occidentale, cette organisation générale bénéficiait fréquemment de deux accès autonome, à des niveaux différents, qui résultaient de l'implantation caractéristique sur versant.

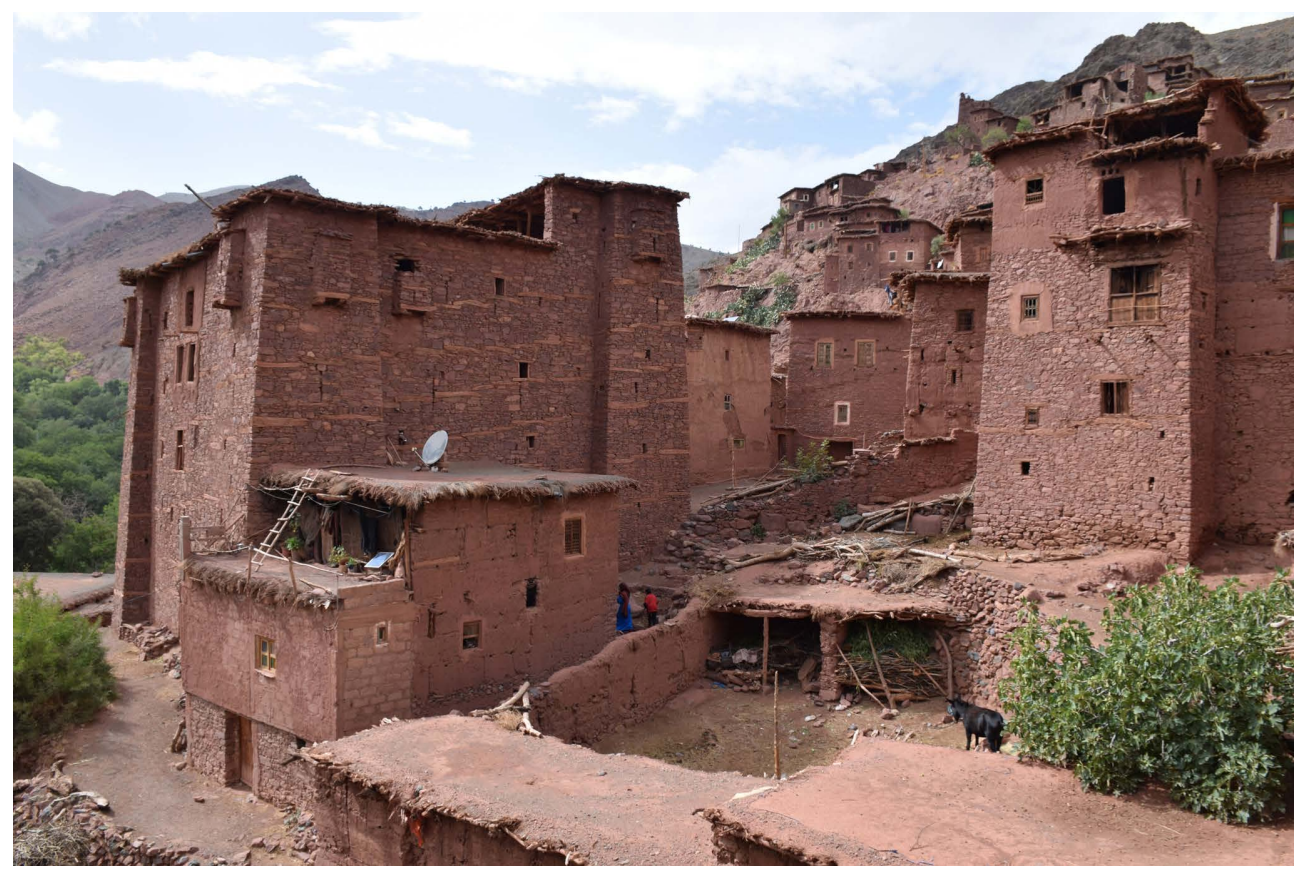

Fig. 3

\section{1. Magdaz dans le Haut Atlas central (tribu Fetouaka)}

C'est cette solution de base que nous rencontrons généralement dans la région centrale du Haut Atlas où, parfois, les édifices les plus anciens ont été transformés jusqu'à avoir quatre ou cinq étages. Afin de comprendre les caractéristiques de cette typologie, il importe de décrire l'un de ces bâtiments que nous avons pu relever dans le village de Magdaz, dans le Haut Tassaout (Fig. 3). Le rez-dechaussée, semi-enterré, contient les dépendances pour le bétail, se prolonge sous l'édifice contigu pour constituer un grenier à foin. L'entrée de l'habitation 
proprement dite, située dans la partie postérieure, était liée aux escaliers qui, en volées superposées, donnaient accès aux divers étages. Le deuxième de ces étages accueille des espaces plus nobles, correspondant à la salle de réception (avec les peintures caractéristique des murs et du plafond) et à la cuisine (avec souvent une fenêtre à croisée). Les autres pièces (situées dans les étages inférieur et supérieur) étaient utilisées de façon saisonnière, dans les étages plus bas pendant la saison froide et dans les étages plus hauts pendant la saison chaude (Fig. 4). C'est dans ce cas que la couverture en terrasse devient un prolongement de l'habitation où, hormis pendant la saison des neiges et des pluies, on pouvait y réaliser diverses tâches domestiques et sécher les récoltes. D'ailleurs, cette caractéristique se retrouve dans tout le Haut Atlas avec très souvent la présence supplémentaire d'un auvent sur la terrasse, comme on le remarque à Magdaz.

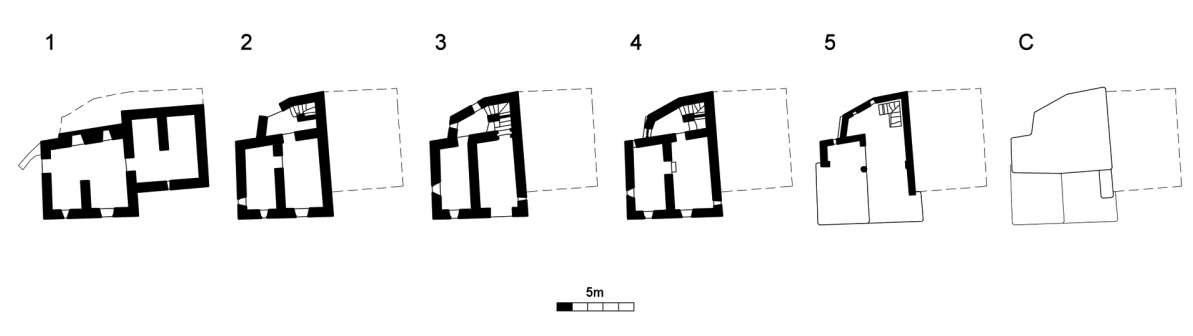

Fig. 4

Au niveau du type de construction, l'architecture de village adopte des solutions diversifiées qui, très souvent, se matérialisent dans la élévation graduelle des édifices grâce à la combinaison du pisé, de murs en adobes et de murs en pierres de schiste et de grès (Fig. 3). Dans certains cas, la technique la plus recherchée est celle de la maçonnerie de schiste liée au bois de genévrier, destinée à renforcer les angles et les parements extérieurs, technique qui, bien que présente dans quelques habitations, apparait surtout liée aux greniers de familles de haut lignage qui structuraient de loin en loin l'ensemble bâti du village.

\section{2. Imeguiss, dans le Haut Atlas central (tribu Fetouaka)}

Le territoire de la tribu Fetouaka, où se trouve le village de Magdaz, constitue aussi un bon sujet d'étude pour appréhender la diversité typologique, en lien avec la caractérisation des diverses sous-unités de paysage d'altitude. Du point de vue du peuplement, cette diversité repose sur la relation du village 
(douar) avec les établissements secondaires (azib) d'altitude, ce qui entraîne des répercussions non seulement au niveau du type de construction mais aussi dans la propre typologie des habitations. En montant dans les villages d'altitude, la construction des murs en terre disparaît, cette technique étant réservée au revêtement de la terrasse. Les constructions temporaires, liées à la transhumance des grands troupeaux de petit bétail, constituent le modèle fondamental des azib comportant des structures de pierre et de bois de genévrier dont les troncs sont amplement intégrés aux murs, en particulier dans la construction du mur extérieur d'enceinte de l'enclos pour le bétail.

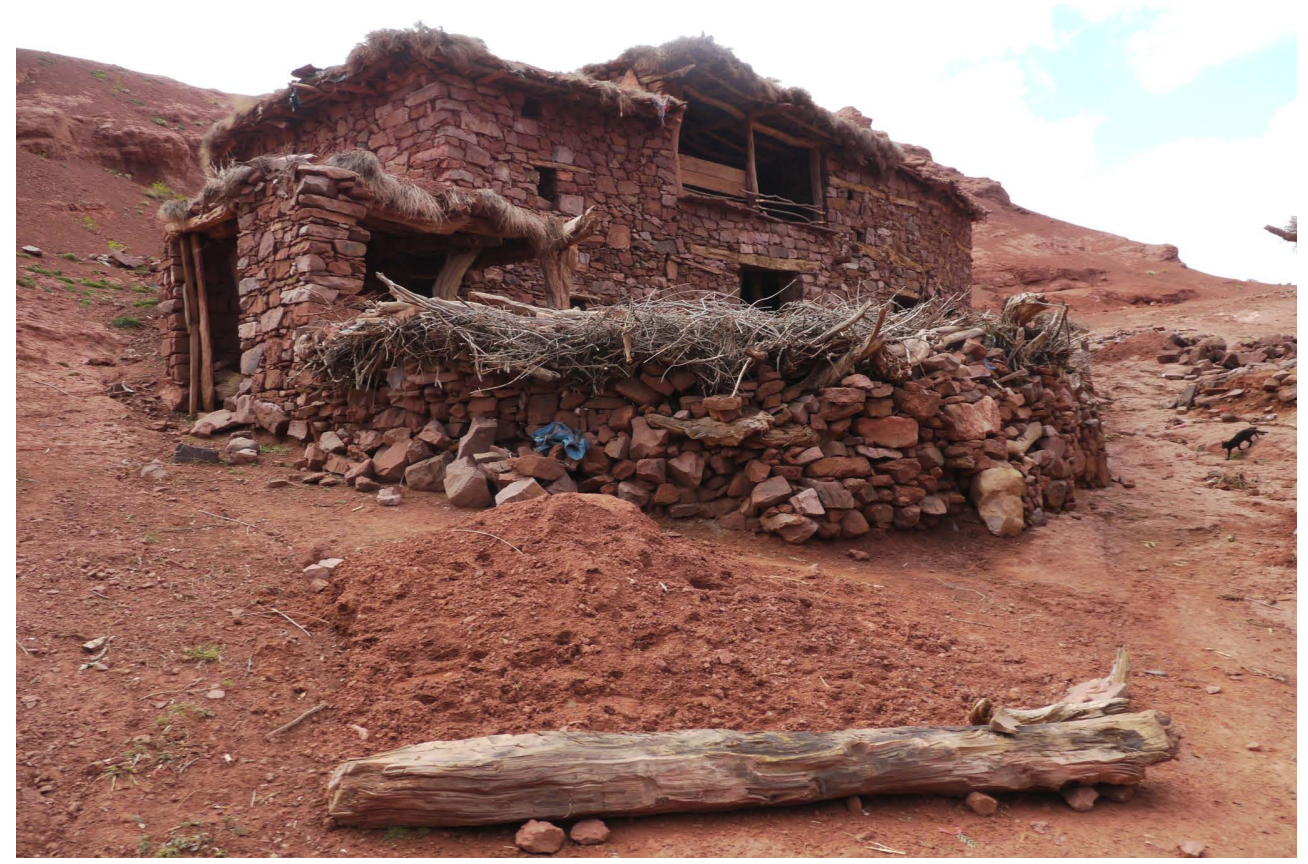

Fig. 5

Dans quelques cas, certaines des constructions d'altitude finissent par devenir une habitation permanente, comme cela est arrivé à Imeguiss situé à près de deux kilomètres au sud de Magdaz (2100 et 1961 d'altitude respectivement). Un des édifices permanents les plus anciens de ce noyau résulte d'une mixité typologique du douar et de l'azib dans cette région (Fig. 5). Le rez-dechaussée rappelle l'organisation des logements temporaires d'altitude, dans laquelle diverses dépendances pour le bétail (avec pied-droit relativement bas et sol non travaillé) se terminent au sud par un enclos délimité par un 
mur au tracé curviligne. L'étage supérieur reprend une solution présente dans de nombreuses habitations de Magdaz, à savoir que le porche est intégré non pas au niveau de la terrasse mais au niveau de l'étage principal de l'habitation, ce qui permettait l'accès aux divers compartiments (Fig. 6).
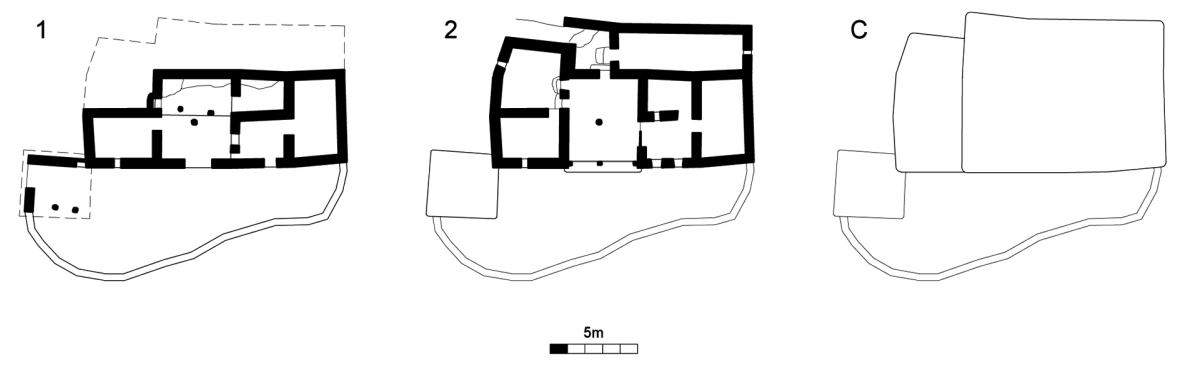

Fig. 6

\section{3. ksar de Tazrouft, Sidi Hamza, dans le Haut Atlas oriental (tribu Aït Izdeg)}

En nous rapprochant de la Jbel Ayachi (3757 mètres) et de Midelt, dans la région orientale du Haut Atlas, nous enregistrons une altération graduelle mais profonde de l'habitat qui, bien que plus évidente au niveau du peuplement ou de son assise, marque également de façon visible la propre typologie de la maison. Les villages de montagne, qui ici sont plus espacés entre eux, acquièrent parmi les populations la désignation de ksar, confirmant par là une organisation plus défensive et présentant d'évidentes analogies avec les villages fortifiés des régions plus méridionales des vallées du AntiAtlas. La ksar de Tazrouft, dans le Sidi Hamza, est l'un des exemples les plus intéressants de cette typologie, caractérisé par le dessin du promontoire où il s'est implanté et par la grande densité de l'ensemble édifié (Fig. 7). Cet ensemble définit une délimitation nette, avec peu d'ouvertures (qui depuis ont été ouvertes en plus grand nombre) et le caractère défensif original est accentué par la disposition des ruelles, ce qui restreint au minimum le nombre de portes de la ksar ouvertes vers l'extérieur. Sur le plan constructif, ces édifices confirment l'usage abondant du bois de cèdre, caractéristique des villages plus anciens de la région, qui soulage les murs de pisé, d'adobes ou de pierre d'une bonne partie du poids apporté par les sols et les terrasses, comme cela est spécialement évident dans les portiques des ruelles couvertes (Fig. 8). 


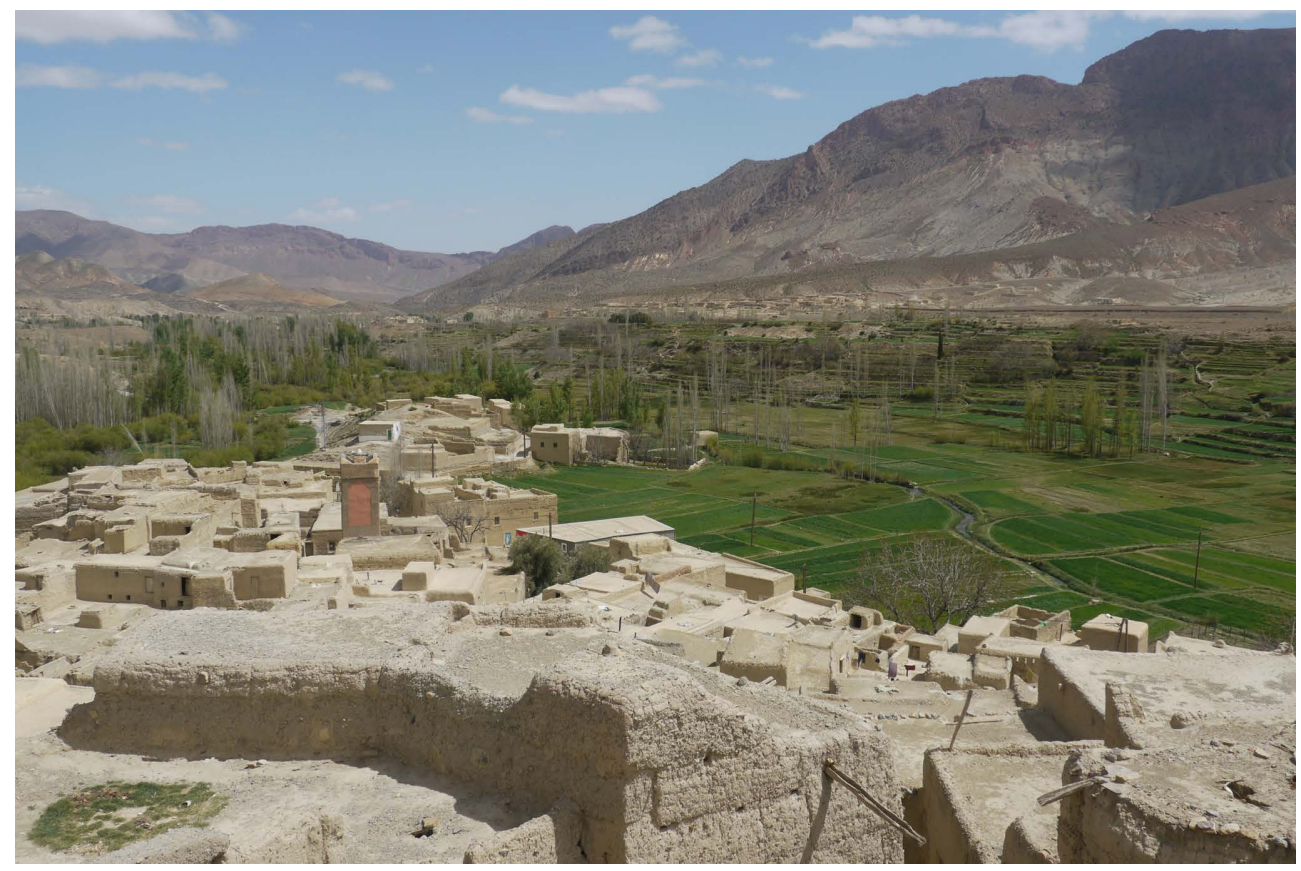

Fig. 7

La maison relevée dans le cadre de notre recherche est cohérente avec ce principe d'organisation, ce qui révèle la densification constructive à l'intérieur de l'ancien ksar de Tazrouft; elle est située sur une petite place, au centre du noyau urbain, vers laquelle convergent diverses ruelles couvertes. L'édifice présente une façade de deux étages, qui combine une large porte au rez-de-chaussée avec une petite fenêtre à l'étage supérieur. Cette composition, très fréquente dans les ksour de la région, révèle l'organisation interne de l'édifice, distinguant le rez-de-chaussée destiné aux animaux et au fourrage de l'étage supérieur, affecté à l'habitation proprement dite. En terme de typologie, l'option d'une unique porte d'entrée est combinée à la proximité d'un escalier qui, d'une certaine façon, permettait d'avoir deux accès autonomes aux deux niveaux (Fig. 9).

Un second aspect fondamental de cette typologie est à mettre en relation avec le fait qu'une unique chambre de grande dimension (avec les diverses occupations domestiques y compris un foyer) occupait la plus grande partie de l'étage supérieur. On retrouve cette caractéristique dans d'autres ksour de la région, comme par exemple, au nord, sur le versant opposé de la Jbel Ayachi (Peyron, 1976: 336) ou dans la vallée d'Outat (Gil Piqueras, 2014: 
193-194). Cependant dans le cas qui nous intéresse, une seconde pièce, qui servait de cuisine, a été installée au-dessus de l'entrée, bénéficiant ainsi de lumière naturelle et d'aération grâce à la présence de l'unique fenêtre de la maison. Cette organisation permettait une utilisation plus flexible de l'espace, considérant non seulement les questions de genre mais aussi les changements saisonniers. A ce sujet, il convient de rappeler que, en dehors de la saison froide, l'étage d'habitation était complété par la terrasse qui, selon les caractéristiques urbaines de la ksar, possédait ici une importance encore plus forte que dans les autres régions du Haut Atlas, car elle était destinée à plusieurs usages différents.

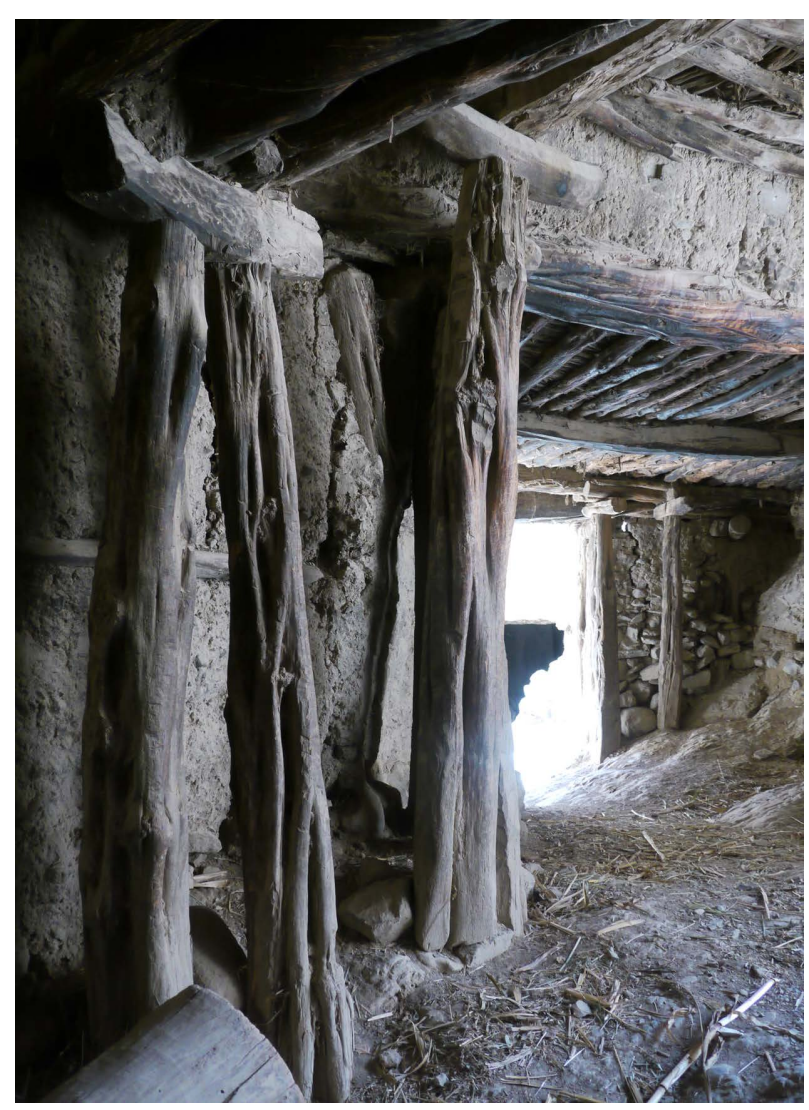

Fig. 8

La distribution interne des divers espaces que nous avons vus rappelle une organisation tripartite, déjà étudiée auparavant pour l'habitat des ksour de la région (Gil Piqueras, 2014: 192-194). Le rez-de-chaussée est caractérisé par une pente prononcée en raison de l'adaptation au terrain; il était constitué par un bloc entrée/escalier (avec l'existence d'un étage inférieur, sous la cuisine, utilisé comme grenier) et par un espace construit en adobes pour les animaux et le fourrage. Cette foisci, le niveau supérieur est organisé en demi-étages: l'espace principal, à un niveau plus bas, permettait ainsi l'accès à un autre espace à travers une entrée qui a été murée par la suite. Cette maison se trouve aujourd'hui inoccupée et dégradée, ce qui confirme la tendance actuelle d'un dépeuplement progressif de la ksar. Ce 
processus est associé au développement d'une couronne externe du peuplement qui, en termes architectoniques, résulte de la supériorité de la maison à un niveau, souvent entourée d'une cour extérieure pour le bétail.
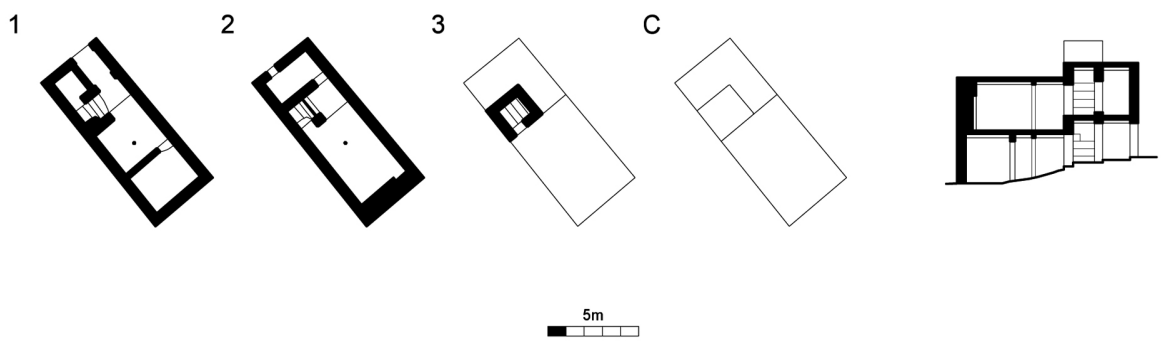

Fig. 9

\section{4. Aït Imi, Tabant, dans le Haut Atlas central (tribu Aït Bougmez)}

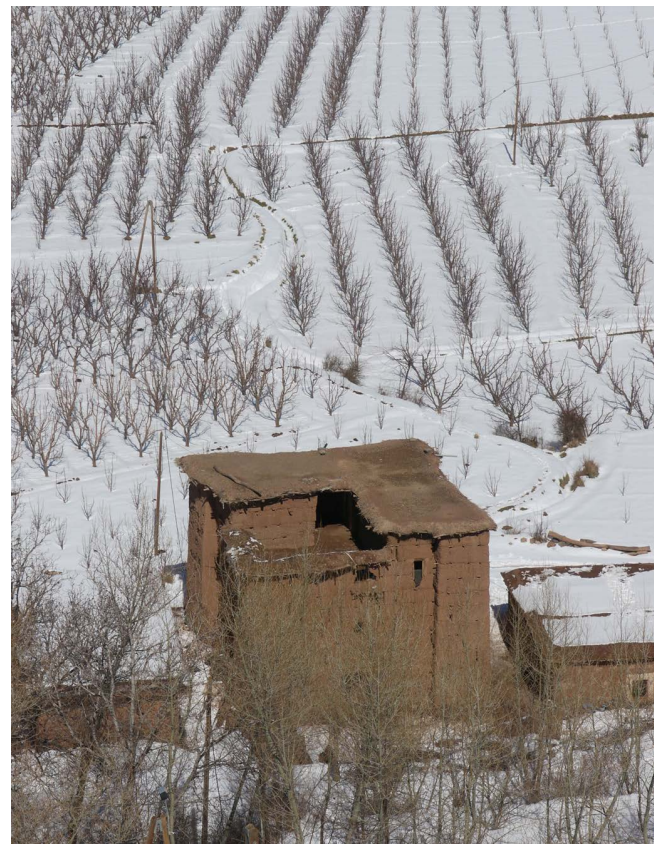

Fig. 10
Outre l'habitation des ksour, la prépondérance de la stratégie défensive est aussi évidente dans d'autres typologies, comme le tighremt. L'expression tighremt renvoie à une construction fortifiée (parfois avec des tours dans les coins) possédant en général une porte unique et étant pratiquement dépourvue de fenêtres (cf. Laoust, 1920), construction qui, selon différents exemples régionaux, peut correspondre soit à un grenier collectif ou un grenier de lignage, soit à une ancienne habitation d'un propriétaire plus connu. Dans le Haut Atlas central, et, plus particulièrement, dans la vallée des Aït Bougmez, l'aspect, la morphologie et l'organisation de 
ces habitations résultent, à partir de la fin du XIXe siècle, d'une influence évidente des greniers fortifiés qui constituent, presque toujours, les structures les plus anciennes.
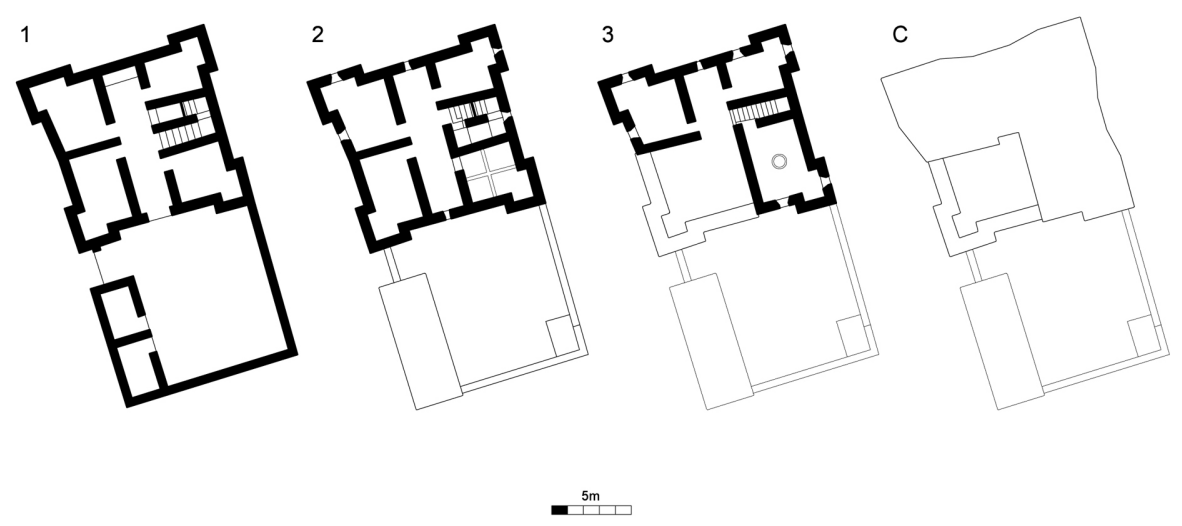

Fig. 11

Ce qui est le cas avec l'édifice relevé dans le village de Aït Imi, près de Tabant (Fig. 10). Au plan rectangulaire, avec quatre tours dans les angles, correspond une organisation interne tripartite, avec un espace intermédiaire de distribution que l'on retrouve à tous les étages (Fig. 11). Cette organisation est très semblable à l'une des solutions types des greniers de lignage que nous avons relevée à Magdaz. La différence fondamentale réside dans la construction des tours: alors que dans le grenier elles constituent des structures effectives de plan quadrangulaire, dans le cas présent elles sont réduites à un simple saillant sur le mur externe de l'édifice. Ainsi, au-delà de l'importance conférée au caractère défensif, cette solution privilégie une dimension de représentation qui la distingue, dans le cadre socioculturel, de l'architecture domestique courante de la région.

Une grande partie des habitations incluses dans cette typologie présente un espace extérieur contigu et clôturé, que l'on devait traverser pour accéder à l'habitation. Servant aussi de parc à bestiaux, cet espace intègre, dans le cas présent, deux dépendances pour le bétail plus petit et un porche pour le four à pain. Au niveau de l'organisation des différents usages dans l'espace intérieur, cette habitation présente une structure claire, qui confirme certains 
lieux communs que nous venons de décrire pour d'autres villages. Le rez-dechaussée est ici aussi réservé au fourrage et aux dépendances pour le bétail, grand et petit; il comprend aussi une mangeoire, au fond de l'espace central de distribution, où l'on pouvait attacher une mule.

Le premier étage constituait l'habitation proprement dite et se composait de trois chambres et d'un cellier. L'importance de l'espace central de distribution se maintenait, espace dans lequel on effectuait aussi les tâches domestiques les plus diverses, y compris faire du feu pendant la saison froide. Le dessin de l'escalier et l'intégration de la porte permettaient de protéger cette partie de l'habitation du regard des visiteurs accédant au dernier étage qui constituait l'aire de réception et d'hospitalité. La maison comprenait ici la cuisine et deux salles de réception séparées par un petit vestibule, qui ensemble encadraient la terrasse tournée vers les champs irrigués de la vallée.

Comme nous l'avons noté précédemment, la difficulté du maintien de ces structures provient, très souvent, de leur conversion en dépendances et dans la construction proche d'une nouvelle habitation (cf. Huet et Lamazou, 1988: 18-19). Dans le cas présent, cette transformation a eu lieu à une période très récente, suite à l'ouverture d'une seconde porte dans le parc à bestiaux pour établir une liaison plus facile vers la nouvelle habitation à un étage qui, dans tous les cas, maintient l'organisation interne tripartite et le recours au pisé dans la construction des murs extérieurs et intérieurs.

\subsection{Bin el Ouidane, dans la transition des régions centrale et orientale du Haut Atlas (tribu Aït Bouzid)}

Bien que le peuplement concentré des villages constitue la forme d'occupation prédominante du Haut Atlas, on peut rencontrer une certaine dispersion dans certaines sous-unités. C'est ce que l'on constate dans la transition entre les régions centrale et orientale, transition marquée, spécialement sur le versant nord des montagnes de Beni-Mellal, par l'apparition d'un peuplement dispersé de maisons isolées ou de hameaux comprenant peu de foyers. Outre l'étagement des pâturages, ce peuplement est à mettre en relation avec la dispersion des parcelles agricoles également. Localisées sur des versants au relief modéré, ces parcelles sont caractérisées par l'existence de vergers en terrain non irrigué et de céréales sous couvert, terrain marqué par des lignes discontinues de levées de pierre sèche rudimentaires, destinées à contrarier l'érosion et à retenir l'humidité bien rare du sol. Malgré la transformation significative du peuplement, les greniers de lignage, que nous avons signalés pour les zones des tribus Fetouaka et Aït Bougmez, maintiennent 
ici leur importance, non seulement dans quelques villages existants, mais aussi dans des zones davantage caractérisées par des constructions. Dans le tiers intermédiaire de ces montagnes, nous pouvons rencontrer soit un petit logement (avec cuisine et chambre) associé à l'enclos du bétail (ammazi), avec une fréquente occupation saisonnière et constituant le point de départ en été vers les pâturages d'altitude, soit une habitation d'occupation quasi permanente (taddart) «[...] composée, sur un même niveau généralement, de plusieurs pièces disposées autour d'un vestibule servant d'étable» (Crépeau et Tamim, 1986: 368).

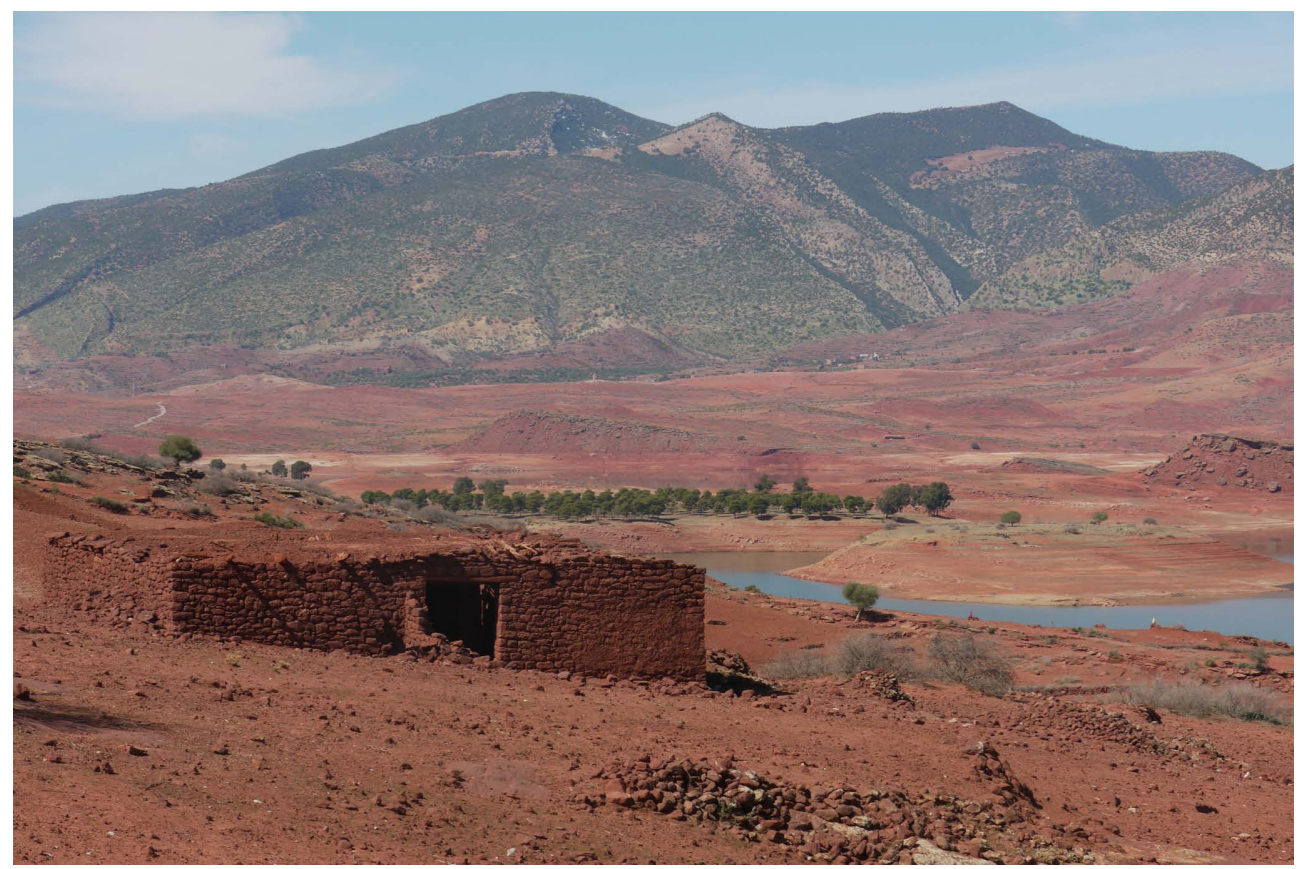

Fig. 12

$C^{\prime}$ est dans cette dernière catégorie que s'inscrit l'habitation relevée sur la rive méridionale du barrage de Bin el Ouidane (Fig. 12) qui permet, bien qu'inoccupée, de recueillir un ensemble de caractères communs aux édifices de cette sous-unité. Il s'agit d'une construction au plan presque carré (Fig. 13), avec un seul étage, une couverture en terrasse et un nombre exigu d'ouvertures, caractéristiques très fréquentes dans les habitations de la région, souvent associées à un enclos délimité par une haie de broussailles. D'une façon générale, ces constructions traduisent, à travers un plan plus 
découpé ou des joints dans les murs de pierre, un processus évolutif et cumulatif de transformation, presque toujours manifeste dans l'architecture des régions de montagne.

1

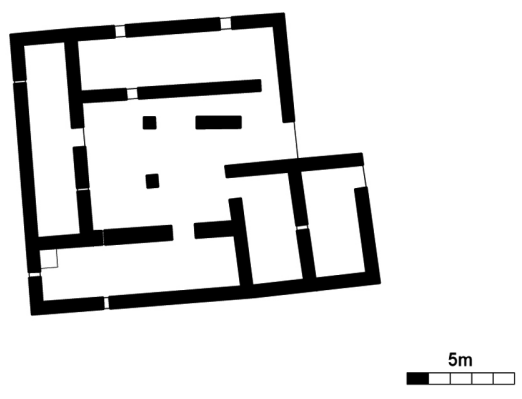

C

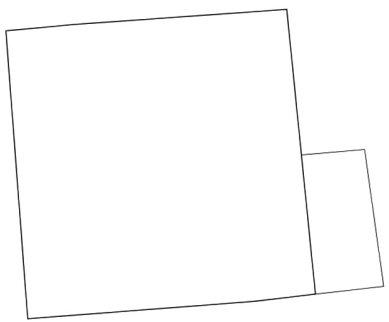

Fig. 13

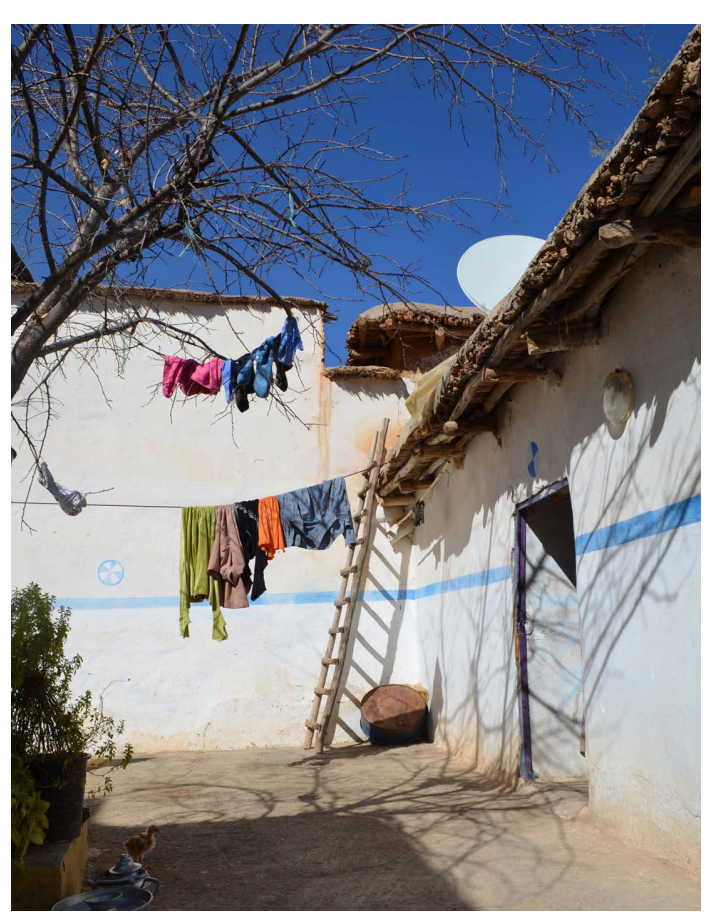

Fig. 14
Dans le cas présent, il est possible de retrouver l'édification originale, constituée par un espace unique qui aujourd'hui correspond au compartiment ouest. Dans une phase postérieure, un second compartiment a été ajouté au sud, les deux délimitant certainement un enclos pour le bétail comme cela est commun dans d'autres constructions de la région. Un troisième moment provient d'une sorte de conversion de l'amazir en taddart, avec l'ajout de deux pièces, au nord et à l'est, et avec l'intégration de la couverture de l'espace central. Enfin, un dernier compartiment fut ajouté sur la façade orientale, dont la dimension fut déterminée par la position de la porte d'entrée 
de l'habitation, donnant un périmètre irrégulier à partir du carré de base. Le plan de cet édifice présente une ressemblance évidente avec celui de la maison patio, typique des régions occidentales du Haut Atlas, qui sera caractérisé par la suite. Cette ressemblance repose sur des critères d'organisation (espace central d'articulation des divers compartiments), de dimension et de proportion (compartiments allongés autour d'un espace central presque carré) et de relation avec l'extérieur (deux fenêtres dans le salon et petites fenêtres ou embrasures dans les autres compartiments).

\section{6. Douar Ifghi, dans le Haut Atlas occidental (tribu Aït Semmeg)}

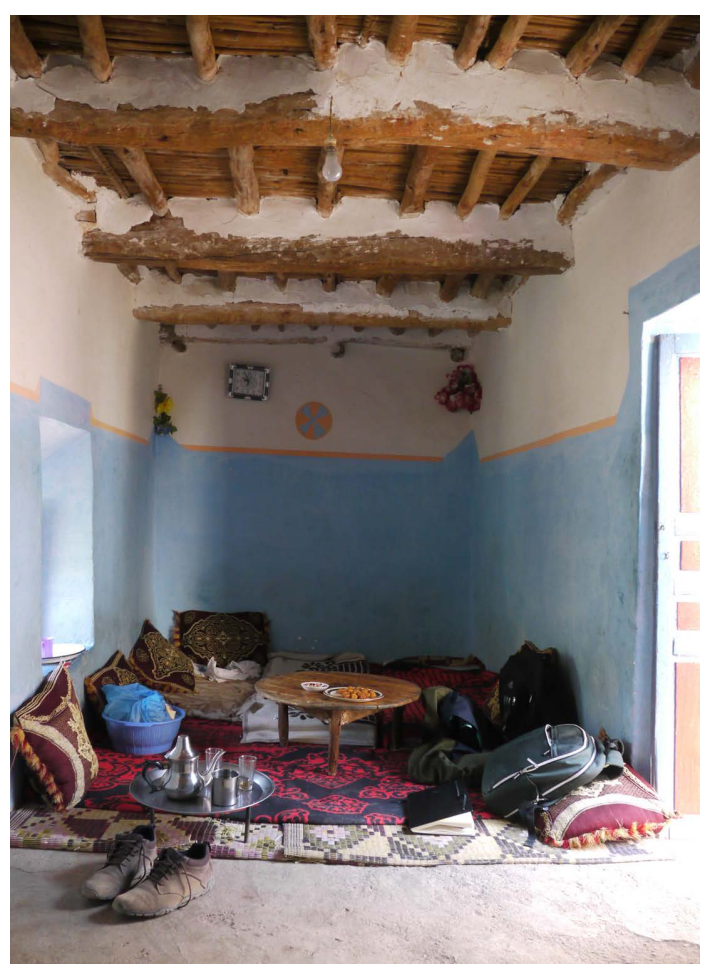

Fig. 15

La maison entourée d'un enclos extérieur non couvert est plus prépondérante dans les sousunités du Haut Atlas occidental, que ce soit sur le versant nord ou que ce soit sur le versant sud. Le type de maison patio caractérise davantage le peuplement à tendance plus éparse, de l'extrémité de la chaîne montagneuse (en particulier l'ouest de la vallée de l'oued Issen, affluent de l'oued Sous) ainsi que les constructions plus denses des noyaux primitifs parfois perchés sur un éperon dominant les champs irrigués. C'est ce que l'on constate, par exemple, dans les villages des versants de l'Argana, creusés par l'oued Taïoukht et ses affluents (sur le versant nord) ou dans les vallées de l'oued Targa (sur le versant sud cette fois). La maison que nous avons relevée dans le village de Douar Ifghi, sur la rive droite de ce cours d'eau, réunit des caractéristiques de la maison patio (Fig. 14), telles que la largeur réduite des compartiments (autour de 2 mètres) (Fig. 15), une certaine hiérarchisation des différents espaces (avec des superficies assez variables), ou bien la liaison, sur le 
même plan horizontal, des diverses chambres autour du patio (Fig. 16). De toute façon, ce dernier aspect est lié avec à une implantation en pente, typique des zones de montagne, provenant aussi de la présence de plusieurs étages construits sur des terrasses successives.

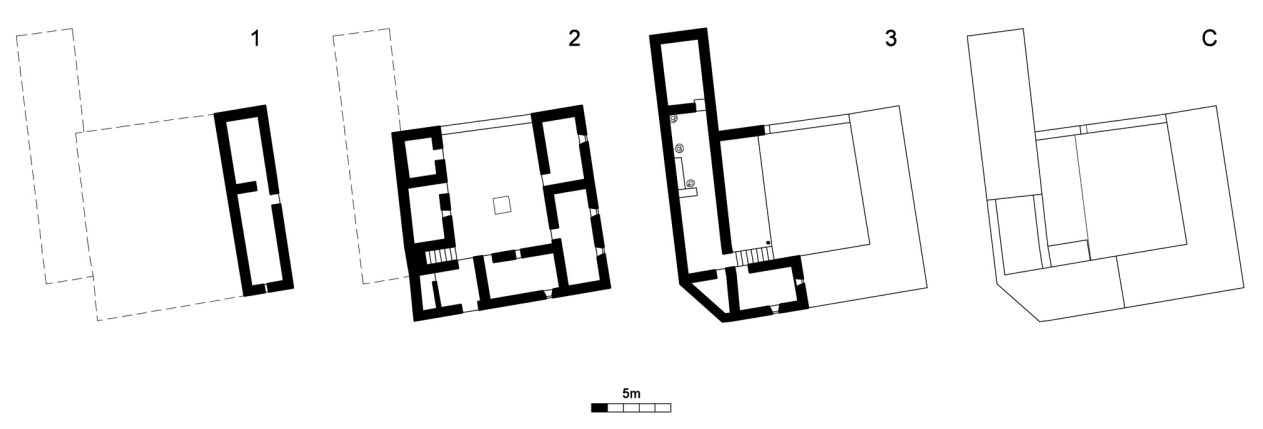

Fig. 16

Le patio constitue l'espace fondamental pour l'articulation des différentes parties de l'habitation, non seulement à l'intérieur mais aussi pour les terrasses extérieures, accessibles par des escaliers en maçonnerie ou en bois. Son occupation est variable selon les saisons et aussi tout au long du jour, car on y retrouve l'alternance caractéristique de domination, de la part de l'homme ou de la femme, que l'on a pu constater dans d'autres zones du Maroc (cf. Delaigue \& El Hraiki, 2015: 233). Dans le cas présent, le patio a les dimensions approximatives de 7,50 sur 6 mètres, ayant en son centre un petit prunier. On remarque également les dimensions évolutives de ce type, le patio pouvant dans ce cas être délimité sur trois côtés seulement par les différentes salles (pour dormir et pour emmagasiner), et aussi par le salon sur l'aile orientale (avec deux fenêtres ouvrant sur la vallée), par un petit vestibule d'entrée sur l'aile sud (à partir duquel on accédait également aux latrines) et par une petite cuisine sur l'aile nord. En outre, l'habitation comprend également, à l'étage supérieur, la chambre principale (avec une fenêtre ouverte au-dessus de la porte d'entrée), un porche (avec des foyers et une meule destiné à l'extraction de l'huile d'argan), le cellier et le bain, tout comme on trouve, à l'étage inférieur, une écurie et un grenier à foin dépourvus de toute communication interne avec le reste de l'habitation. 


\section{7. Douzrou, dans le Haut Atlas central (tribu Guedmioua)}

L'organisation de l'habitation autour d'un enclos découvert peut, cependant, provenir de solutions plus complexes que celle que nous venons de décrire. Dans quelques cas, la séparation entre les espaces d'habitation et ceux des dépendances pour le bétail, qui existe dans la maison de Douar Ifghi, est renforcée par la présence d'un second mur d'enceinte qui délimite un espace ayant pu servir de bergerie ou d'étable. Dans les maisons plus importantes, on peut même constater un certain démembrement de l'habitation en divers espaces clos, qui révèlent différentes étapes dans la construction. On peut ainsi identifier un nombre très significatif de variantes du type d'habitation organisé autour d'un espace central découvert.

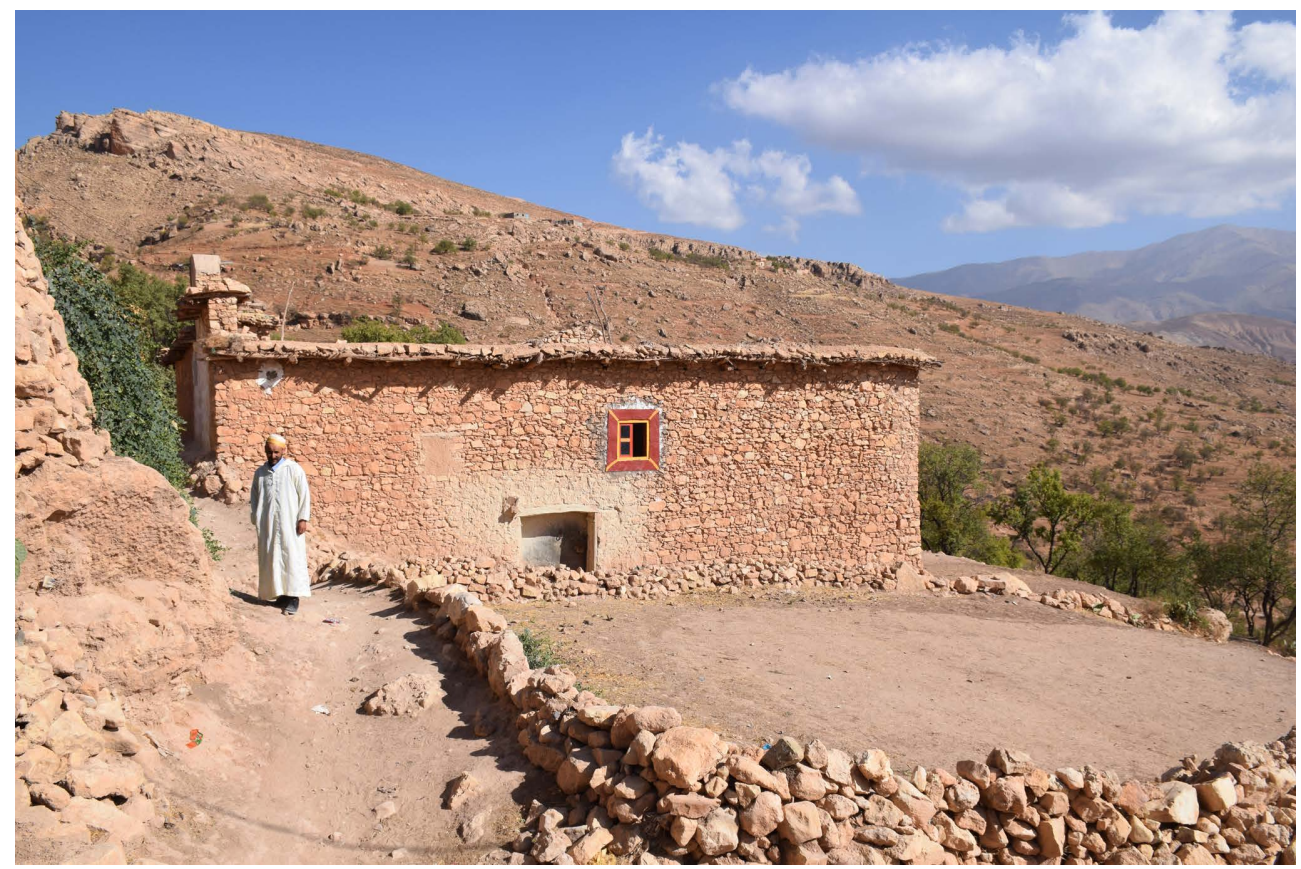

Fig. 17

Un dernier exemple qu'il nous importe de présenter, à ce sujet, est une habitation située dans le village de Douzrou, implantée sur le versant occidental de la Jbel Taourirt, à environ $35 \mathrm{~km}$ au nord de Douar Ifghi (Fig. 17). Dans ce cas, l'édifice est organisé autour d'un espace clos, qui servait de parc à bestiaux, à partir duquel on accédait à des dépendances destinées 
au bétail et aux fourrages, dans l'étage inférieur (Fig. 18). Autrement dit, en gardant la dissociation des dépendances pour le bétail, dans l'étage inférieur, et la maison proprement dite, dans l'étage supérieur, l'espace central à découvert apparaissant ici relié au premier (et non au second, comme il advient à Douar Ifghi) (Fig. 19). La liaison entre les divers espaces de la maison est de ce fait organisée à partir d'un couloir en forme de L (Fig. 20), avec la porte d'entrée et le salon à une extrémité, les diverses chambres dans la partie intermédiaire, la cuisine et l'escalier d'accès à la terrasse supérieure à l'extrémité opposée.

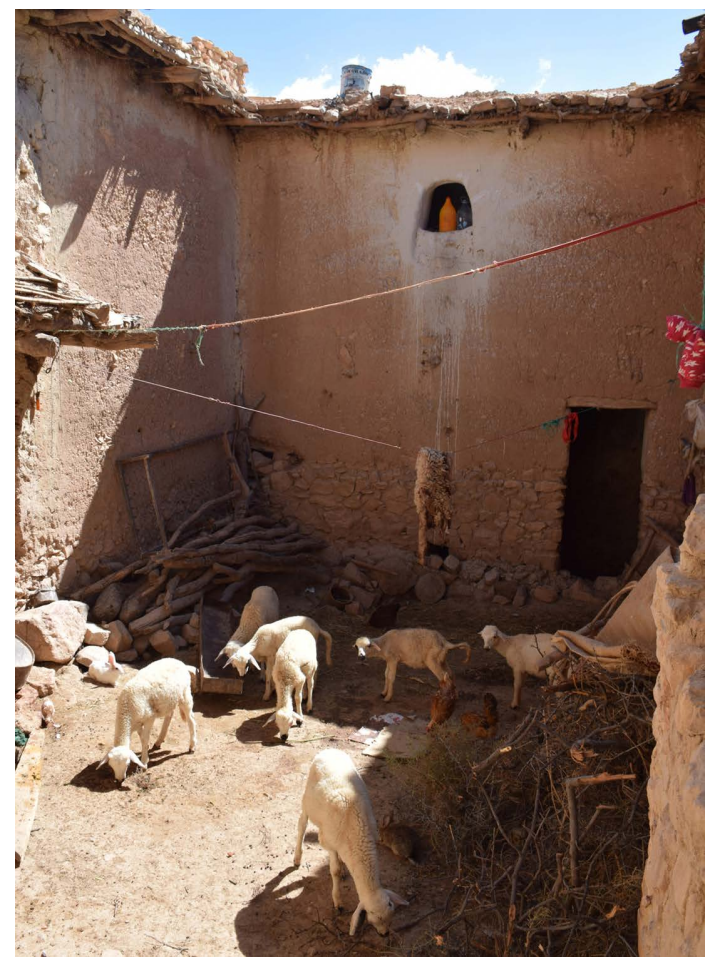

Fig. 18

\section{Conclusion: critères à prendre en compte pour éta- blir une carte typologique}

La caractérisation de l'ensemble des habitations relevées dans différentes sousunités géographiques du Haut Atlas, parallèlement à d'autres cas d'étude traités par d'autres auteurs, rend évidente la grande diversité de l'architecture domestique dans cette chaîne de montagne. Dans le cas présent, on a cherché à reconnaître cette diversité en restreignant l'objet de l'étude à l'habitation permanente, sans tenir compte donc des habitats temporaires et les structures associées à la petite ou grande transhumance. En termes génériques, il est possible de considérer deux mo-

dèles fondamentaux d'organisation de l'habitation qui ont acquis une expression diatopique: le premier est à mettre en relation avec ce que l'on pourrait désigner par «maison-bloc», où les différents espaces d'habitation constituent un volume fermé, dans lequel on trouve différentes solutions d'articulation des compartiments (groupe d'escaliers, espace principal, couloir partiel, etc.); le second correspond à ce que nous pour- 
rions désigner «maison-espace central», autour duquel les divers compartiments de l'habitation sont disposés, intégrant des solutions plus complexes que la maison patio. Bien que la répartition géographique de ces deux modèles dépend aussi du critère d'altitude (en distinguant les tiers inférieur et médian du tiers supérieur), la détermination des aires affectées à chacun d'eux est très claire au niveau des grandes sous-unités régionales, en distinguant le Haut Atlas oriental et central, où domine la «maison-bloc», du Haut Atlas occidental où domine la «maison-espace central».

1

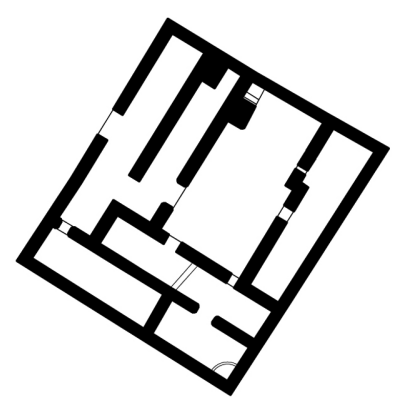

2
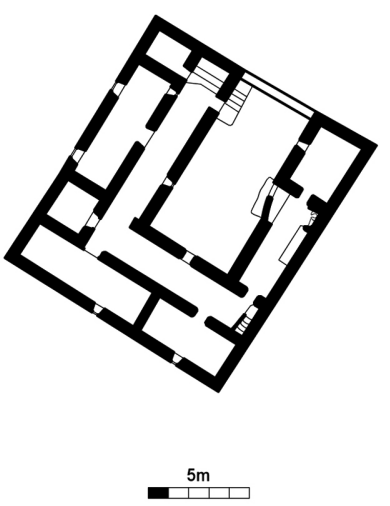

C

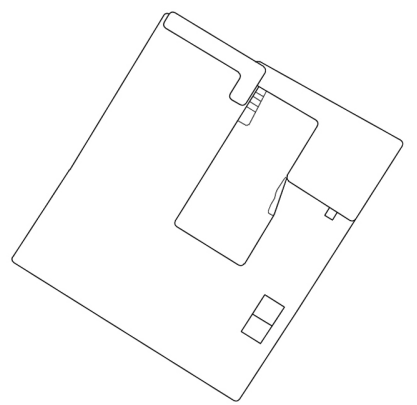

Fig. 19

La poursuite de cette recherche permettra d'intégrer ce premier niveau de différenciation dans le débat mettant en opposition le modèle de la maison berbère et le modèle de la maison arabe. Et aussi parce que quelques-unes des variantes de la «maison-bloc» rappellent la grande cuisine et l'habitation à espace unique ayant une superficie notable. De toute façon, une des caractéristiques les plus intéressantes de l'architecture domestique de la région provient de la grande variété typologique et des formes de mixité que l'on trouve dans les deux modèles en question. Il importe de distinguer, à ce propos, les thèmes suivants: une entrée commune ou une entrée séparée de l'habitation et des dépendances du bétail; l'utilisation saisonnière ou permanente des divers espaces; la diversité de l'espace central autour duquel s'organise l'habitation qui 
pourrait aller du patio ou du parc à bestiaux jusqu'à l'espace couvert avec ou sans lucarne; le caractère défensif ou le caractère représentatif de l'architecture; l'intégration dans l'ensemble de l'édifice, depuis les géométries les plus complexes et enchevêtrées, propres du centre des villages, jusqu'aux solutions plus dissociées dans la périphérie; ou le lien de la construction avec les différents modèles de peuplement (des villages et des ksours, à la dispersion presque toujours relativement récente).

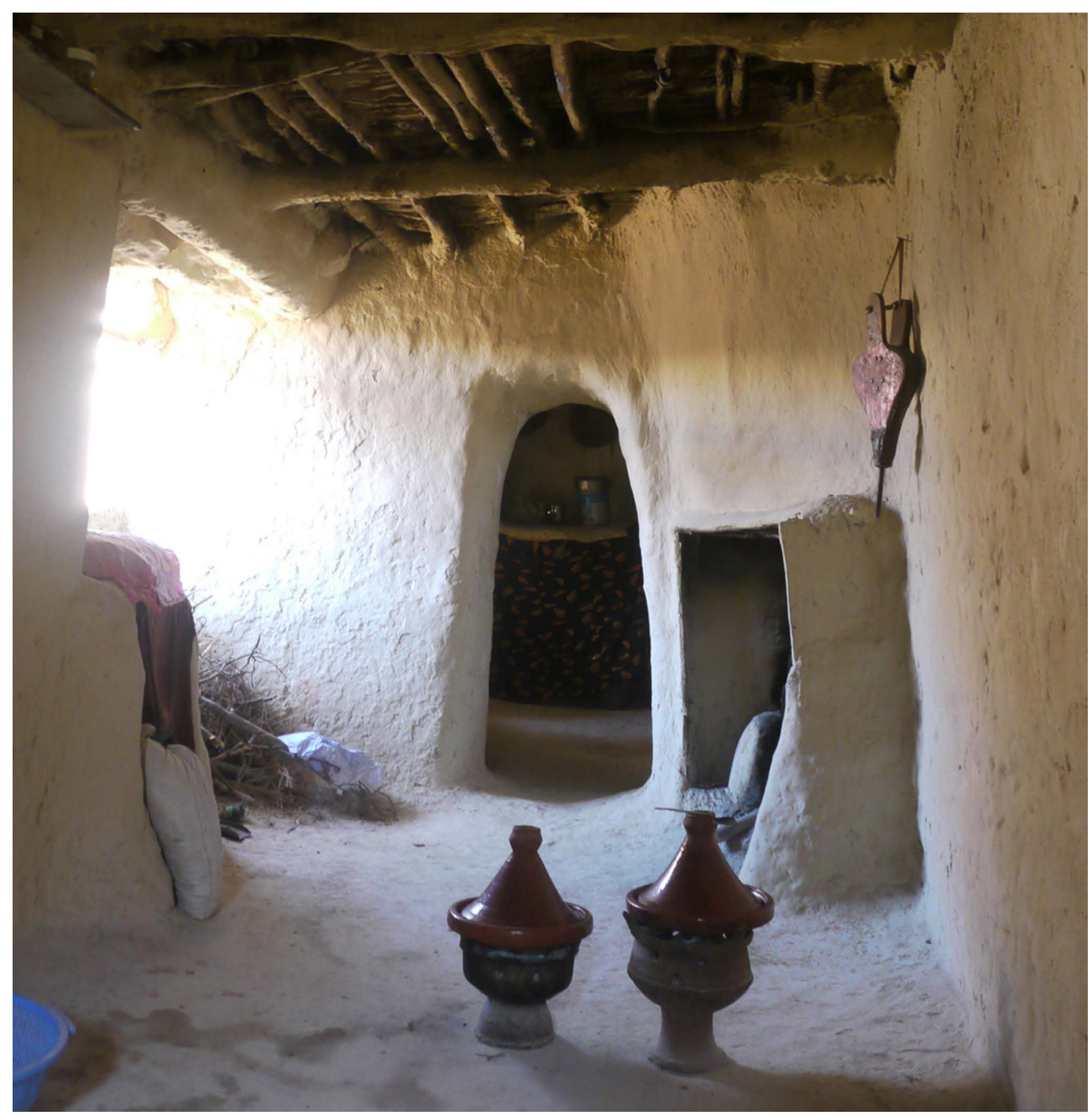

Fig. 20 
La diversité de l'architecture du Haut Atlas provient avant tout du propre support géologique. Dans de nombreux endroits, dans les diverses sousunités géographiques de la zone étudiée, l'architecture de terre présente aussi une importance décisive. Outre son utilisation privilégiée sur les terrasses, les murs de pisé ou en adobes caractérisent une grande partie des villages du Haut Atlas, car ils permettent, comme on le sait, un procédé de construction plus rapide et économique par rapport aux murs de pierre. De toute façon, comme l'a noté Terrasse (2010: 47), la pierre est le matériel le plus adéquat à ces zones montagneuses, en particulier là où les conditions naturelles sont plus difficiles. C'est ce qui se passe en haute montagne, dans les zones de plus forte influence atlantique, ou sur les versants les plus battus par les pluies de l'ouest. De fait, dans n'importe quelle des sous-unités étudiées, il est possible de rencontrer des maisons de terre protégées, en particulier dans le quart occidental, par d'autres matériaux, depuis le revêtement de planche de bois de cèdre, à l'est, jusqu'à la maçonnerie en pierre à l'ouest. La diversité est ainsi, par définition, est une des caractéristiques fondamentales de l'architecture vernaculaire, grâce au recours essentiel aux matériaux locaux, également évident dans le recours au bois. Les différentes expressions de cette architecture, de pierre et de terre, intègrent systématiquement les diverses espèces de la flore présentes, selon les caractéristiques des bois respectifs, que ce soit dans les structures ou dans les décorations. Les différentes typologies d'habitation, associées aux différentes sousunités de paysage de la haute montagne de l'Atlas, utilisent de façon privilégiée, de l'est vers l'ouest et selon l'altitude, le bois de cèdre, sans le Haut Atlas oriental, le bois de genévrier thurifère dans le Haut Atlas central et occidental et, dans cette dernière région, à des niveaux moyens, les bois d'arganier et de dattier, qui sont surpassés, quoique selon des façons diverse, par le bois de peuplier blanc abondant dans la forêt longeant les vallées jusqu'à près de 2000 mètres d'altitude. Dans chaque région, on cherchait à tirer parti des caractéristiques particulières de chaque espèce pour la fabrication non seulement des revêtements (en distinguant éléments primaires, secondaires et bardage), mais aussi des colonnes, spécialement dans les porches des habitations et dans les abris temporaires des grands troupeaux. Dans ce contexte, on remarque particulièrement les ksour du Haut Atlas oriental, caractérisés par le recours abondant au bois de cèdre, surtout dans les structures complexes des portiques, complémentaires des murs de pierre et encore plus de terre. 
La transformation plus ou moins profonde qu' ont subie ces établissements de la zone étudiée, dans les dernières décennies, a achevé de diversifier cette architecture, tant au niveau des procédés de construction (avec l'introduction de matériaux industriels) qu'au niveau de l'organisation de l'habitation, avec l'influence croissante de modèles extérieurs. Mais dans la réalité, cette conjoncture est associée à une phase de transition dans laquelle, très souvent, sont combinés différents modes de construire et d'habiter. Le remplacement progressif des solutions traditionnelles par des solutions contemporaines aurait tendance à simplifier et à uniformiser cette architecture. Dans certains cas, cette simplification est déjà bien évidente, en particulier au niveau l'utilisation prépondérante des matériaux de production industrielle, identique dans les divers territoires et n'ayant que peu à voir avec la diversité géographique et celle des paysages du Haut Atlas.

\section{Bibliographie}

Bellaoui, Ahmed (1986), Le Haut-Atlas occidental quarante ans après. Revue de l'Occident musulman et de la Méditerranée, n41-42, pp. 216-233.

Crépeau, Christian ; Tamim, Mohamed (1986), Communautés pastorales et systèmes d'habitat dans le Haut-Atlas de Beni-Mellal (Maroc). Annuaire de l'Afrique du Nord, Tome XXV.

Delaigue, Marie-Christine; El Hraiki, Rahma (2015), Mujeres y casas en el medio rural del norte de marruecos. Una aproximación etnografica. In Diez Jorge, M. Elena; Navarro Palazón, Julio (ed.), "El espacio doméstico en la Península Ibérica medieval. Sociedad, familia, arquitectura, ajuar (conference, 2013)". Madrid: Sílex. pp. 225-236. Gil Piqueras, Teresa (2014), Arquitectura de tierra en el Alto Atlas. Del Oasis de Mdagra al Valle del Outat. Tesis Doctoral. València: Universitat Politècnica de València.

Huet, Karin; Lamazou, Titouan (1988), Sous les toits de terre, Haut Atlas: éléments d'architecture traditionnelle et décoration picturale dans l'habitat berbère des hautes vallées. Billère: Publi-action.

Laoust, Émile (1920), Mots et choses berbères, Notes de Linguistique et d'Ethnographie, Dialectes du Maroc. Paris: Augustin Challamel.

Ouhammou, Ahmed (2005), Flore et végétation du Parc National de Toubkal (HautAtlas de Marrakech, Maroc): typologie, écologie et conservation. Thèse de doctorat. Marrakech: Université Cadi Ayyad.

Ouhammou, Ahmed; Muller, Serge; M'Hirit, Omar (1996), Impact des activités pastorales sur la biodiversité floristique dans une thuriféraire limitrophe du Parc 
National de Toubkal, Haut-Atlas de Marrakech, Maroc. Acta botanica Gallica, Vol. 143 Issue 4-5, pp. 393-401.

Peyron, Mickael (1976), Habitat rural et vie montagnarde dans le Haut Atlas de Midelt (Maroc). Revue de géographie alpine, tome 64, n³, pp. 327-363.

Terrasse, Henri, (2010) [1938], Kasbahs berbères de l'Atlas et des oasis. Les grandes architectures du Sud marocain. Rabat, Centre Jacques-Berque. 